\title{
Interpretations of autonomous decision-making in antenatal genetic screening among women in China, Hong Kong and Pakistan
}

\author{
Shenaz Ahmed $\mathbb{D}^{1} \cdot$ Huso Yi $\mathbb{D}^{2} \cdot$ Dong Dong $^{3} \cdot$ Jianfeng Zhu ${ }^{4} \cdot$ Hussain Jafri ${ }^{5}$ Yasmin Rashid ${ }^{6}$ Olivia MY Ngan ${ }^{7} \cdot$ \\ Mushtaq Ahmed ${ }^{7}$
}

Received: 4 September 2017 / Revised: 19 December 2017 / Accepted: 23 December 2017 / Published online: 31 January 2018

(c) European Society of Human Genetics 2018

\begin{abstract}
The concept of informed choice for antenatal screening consists of Western ideologies, encapsulating individualistic approaches, and may be valued differently by people from countries with more collectivist cultures. This study aimed to explore perceptions of informed choice in antenatal screening in women from China, Hong Kong and Pakistan. A Qmethodology study was conducted during June 2016 to February 2017, in China (Shanghai and Duyun), Hong Kong and Pakistan (Lahore). A total of 299 women rank ordered 41 statements. Following by-person factor analysis, five distinct viewpoints were identified: choice as a maternal responsibility entrusted to doctors; choice as a shared decision led by the mother; choice as a shared decision led by the partner; choice as a responsibility delegated to the partner and doctors; and choice within a religious discourse. The findings highlight ethical dilemmas for healthcare professionals in facilitating informed choice for antenatal screening where policy and practice guidelines adapt predominantly individualistic approaches. Women's preferences for decision-making with health professionals and/or their partner, with minimal emphasis on individual rights, suggest the need for clarification of the role of health professionals in supporting and facilitating decision-making to enhance women's autonomy. Policy and practice guidelines need to be (re)framed to facilitate decisionmaking processes for antenatal screening using relational approaches to autonomy.
\end{abstract}

\section{Introduction}

Advancements in prenatal genetic screening technologies continue to enable detection of increasing numbers of complex and rare conditions [1], but also continuingly raise issues about informed choice. This concept is

Electronic supplementary material The online version of this article (https://doi.org/10.1038/s41431-017-0091-1) contains supplementary material, which is available to authorised users.

Shenaz Ahmed

s.ahmed@leeds.ac.uk

University of Leeds, Leeds, UK

2 Chinese University of Hong Kong, Hong Kong, Hong Kong

3 Hong Kong Baptist University, Kowloon Tong, Hong Kong

4 Fudan University, Shanghai Shi, China

5 Genetech Laboratory, Lahore, Pakistan

6 Central Park Medical College, Lahore, Pakistan

7 Yorkshire Regional Genetics Service, Leeds, UK internationally recognised as an important aspect of ethical healthcare [2]; informed choice enables autonomous decision-making and is described as being made when the individual has 'an understanding of the options presented and intentional action without undue influence from others' [3], and is made 'in accordance with the decision-makers' beliefs' [4]. Such interpretations of autonomy emphasise the individual's right to make decisions and relate to concepts such as self-determination, independence, and selfsufficiency [5].

This individualistic approach to autonomy helps safeguard patients from healthcare professionals' (HCPs) paternalistic influences and undue pressure from relatives/ others. However, this approach does not take into account that individuals may not view themselves as independent decision-makers [6], or that individuals are socially embedded and their identities are shaped within the context of social relationships and social determinants [7]. The adaptation of informed choice in policy and practice guidelines for antenatal screening in most Western countries consists of Western ideologies of individualism. This is despite calls for a move towards a more nuanced approach 


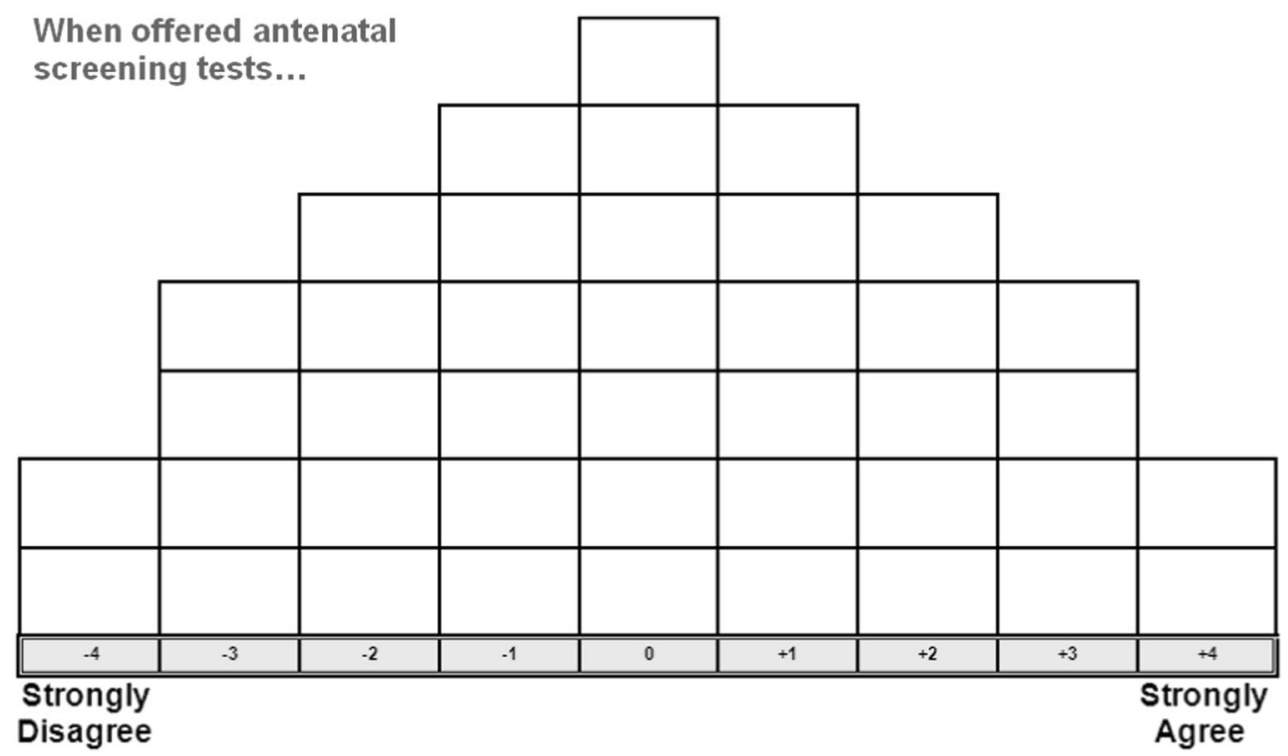

Fig. 1 Q-sorting grid

which incorporates relational approaches to autonomy, to better support decision-making by preventing feelings of helplessness and isolation [8-10]. Furthermore, such individualistic adaptations may be valued differently by people in countries with more collectivist cultures [11]. The rapid implementation of prenatal genetic technologies globally [12], calls for an understanding of informed choice in such cultures.

Relational approaches to autonomy, emphasise 'interdependence' rather than 'independence', where an individual's social environment and personal relationships impact autonomy. Proponents of relational approaches worldwide suggest that involving family and HCPs could enhance individual autonomy [8-10, 13]. This approach tends to predominate in medical decision-making in countries with collectivist cultures $[14,15]$. For example, it is the norm for the husband and family to play a key role in decisionmaking about the health and wellbeing of the pregnant woman and unborn child [16, 17]. For antenatal screening specifically, there is some evidence that Chinese women in Hong Kong (HK) prefer to make decisions with their partner and HCPs, but not other relatives [18]. Nevertheless, there is a paucity of research on views about informed choice for antenatal screening in such countries, hence preferred forms of relational autonomy for decision-making in this context $[18,19]$.

Cross-national research in countries like China, HK and Pakistan could enhance understandings of how people in different cultural, political and religious contexts value informed choice for antenatal screening. Such research is valuable for informing the development of policy and practice guidelines for culturally appropriate implementation of genetic technologies more globally [12], by (a) highlighting the need for policies on informed choice' for antenatal screening, (b) exploring the extent to which different approaches are needed within different countries to support decision-making, and (c) understanding the extent to which current Western adaptations of autonomy could be adapted to support decision-making more globally. Therefore, based on previous research on crosscultural values of informed choice for antenatal screening in the UK [20], this study aimed to explore perceptions of autonomous informed choice for antenatal screening in women from China, HK and Pakistan.

\section{Materials and methods}

This study was carried out in June 2016 to February 2017 using Q-methodology, an approach sensitive to cultural variations, used successfully in cross-national studies [20, 21]. Participants' viewpoints are obtained through their Qsorts. That is, participants read propositions statements (things already written or said about the topic) and rank order them on a Q-sorting grid (see Fig. 1) to show the extent to which they agree or disagree with them [22]. This enables participants to discriminate between statements and think carefully about their views. The set of statements (Qset) for this study had been previously developed for a UK study in English, French, Mandarin, Cantonese and Urdu [20]. This Q-set enabled participants to reflect on their views about how they would prefer to make informed choices about antenatal screening. For details of how the Qset was developed, see the UK study [20]. 
Table 1 Demographic characteristics of study participants $(n=299)$

\begin{tabular}{|c|c|c|c|c|c|}
\hline & & $\begin{array}{l}\text { Shanghai, China; } \\
n=84\end{array}$ & $\begin{array}{l}\text { Duyun, China; } \\
n=85\end{array}$ & $\begin{array}{l}\text { Hong Kong; } \\
n=70\end{array}$ & $\begin{array}{l}\text { Pakistan; } \\
n=60\end{array}$ \\
\hline & & $N(\%)$ & $N(\%)$ & $N(\%)$ & $N(\%)$ \\
\hline \multirow{3}{*}{$\begin{array}{l}\text { Language in which Q-sort } \\
\text { completed }\end{array}$} & Mandarin & $84(100 \%)$ & $85(100 \%)$ & & \\
\hline & Cantonese & & & $70(100 \%)$ & \\
\hline & Urdu & & & & $60(100 \%)$ \\
\hline \multirow[t]{2}{*}{ Participants' education } & $\begin{array}{l}\text { Up to initial education exit } \\
\text { level }\end{array}$ & $3(4 \%)$ & $49(58 \%)$ & $24(34 \%)$ & $16(27 \%)$ \\
\hline & $\begin{array}{l}\text { Above initial education } \\
\text { exit level }\end{array}$ & $81(96 \%)$ & $36(42 \%)$ & $46(66 \%)$ & $44(70 \%)$ \\
\hline Age (years) & Mean (SD) & $31(3.9)$ & $29(4.8)$ & $33(4.3)$ & $28(5.3)$ \\
\hline
\end{tabular}

an China and Hong Kong, this is a 'high school diploma' and 'diploma of secondary education', respectively, at around age 18 and equivalent to UK A'levels. In Pakistan, this is called 'Matriculation level', at around age 16 and equivalent to UK GCSE level

A participant's distribution of statements is known as a Q-sort and is the unit of analysis in Q-methodology. Factor analysis is used to group these Q-sorts based on the similarities and differences in which the statements are ranked by each participant [22]. The factor analytic techniques in Q-methodology correlate people, not variables, hence enable the identification of the variety of views in the sample [23].

\section{Setting}

Following ethical approval by local research ethics committees, the study was conducted in four locations: Shanghai, China; Duyun, China; HK; and Lahore, Pakistan. There are government-funded antenatal screening programmes in HK for Down syndrome, trisomies 13 and 18, and thalassaemia, and in the Punjab province of Pakistan for thalassaemia. Antenatal screening for Down syndrome and thalassaemia has been more widely available in the private sector in China and Pakistan. More advanced prenatal technologies are also continually being introduced.

\section{Participants}

Three locations with Chinese populations were included to capture views of a diverse sample. Although this approach was not logistically possible in Pakistan, we aimed to obtain a diverse sample. Participants were women who had at least one child aged three years or younger to ensure salience of the research topic. Participants could read English, Mandarin, Cantonese or Urdu. Purposive sampling was used to recruit diverse samples by age and education. Participants' religious affiliation was not recorded because most participants in China did not have a religion and most participants in Pakistan would be Muslims. See Table 1 for sample characteristics.

\section{Procedure}

Local researcher assistants (RAs) recruited participants (a) via hospitals, community maternal and child health centres, and other community centres attended by mothers of young children, and/or (b) obtaining a convenience sample by asking colleagues and friends to suggest potential participants. To ensure consistency in data collection, all the RAs used standardised instructions to administer the study (see supplementary information). Q-sorts were administered by the RAs in person and completed individually by participants in convenient locations (health centres, participants' homes or cafes). Participants were provided with a Q-set (41 statements printed on numbered cards) and Q-grid, in Mandarin and Cantonese in China and HK, and in Urdu in Pakistan. Participants read the statements and ranked them from +4 (strongly agree) to -4 (strongly disagree) in relation to the offer of antenatal screening tests. Participants placed each statement on a cell on the Q-grid (Fig. 1). Each participant's distribution of the statements on the Q-grid (the Q-sort) was photographed. Each participant also had a post-sorting interview (audio recorded) to explain their reasons for placing statements on the extreme ends of the Qgrid. These interviews were translated into English and transcribed verbatim.

A total of 299 women completed the Q-sorting procedure (84, 85, 70 and 60 in Shanghai, Duyun, HK and Pakistan, respectively). Data was pooled and analysed to identify views across the four study locations.

\section{Analysis}

Data were analysed using PQ-method [24]. Factor analysis was used to identify clusters of participants with highly correlated Q-sorts (factors), which could then be interpreted as distinguishable viewpoints. Factors were extracted using principal components analysis, which maximises 
Table 2 Factor arrays: scores against each statement by viewpoint

\begin{tabular}{|c|c|c|c|c|c|c|}
\hline No. & Statement & $\mathrm{V} 1$ & $\mathrm{~V} 2$ & V3 & $\mathrm{V} 4$ & V5 \\
\hline 1 & It's best to take one step at a time- - to have the tests and not worry about what could happen & 3 & 3 & 3 & 4 & 0 \\
\hline 2 & It is important for me to think about the challenge of bringing up a child with the condition & 2 & 2 & 1 & 3 & 2 \\
\hline 3 & I think the offer of tests suggests people with these conditions are worth less than others & -2 & -2 & -4 & 0 & -2 \\
\hline 4 & I would worry about the child with the condition being treated badly by society & 0 & 2 & -4 & 3 & 1 \\
\hline 5 & I would look for what my religion says about having such testing & 0 & -1 & -1 & -4 & 3 \\
\hline 6 & I would not discuss testing with anyone because the decision is mine alone & 0 & 0 & -3 & -3 & -3 \\
\hline 7 & I would be angry if I was tested without being asked for my permission & 0 & 2 & 2 & 0 & 2 \\
\hline 8 & Doctors/midwives should give me their professional advice about whether to have testing & 3 & 3 & 3 & 3 & 4 \\
\hline 9 & I would leave the decision about testing to doctors/midwives & 2 & -3 & -2 & 1 & -2 \\
\hline 10 & If lots of other people are having testing, then testing would be fine by me & 1 & 0 & -1 & 0 & 1 \\
\hline 11 & $\begin{array}{l}\text { The decision about these tests is no more difficult to make than routine health tests in pregnancy, such as the } \\
\text { mother's blood pressure or diabetes }\end{array}$ & 1 & 0 & 2 & -1 & 0 \\
\hline 12 & There is no decision for me to make because the tests are just part of good care for pregnant women & 4 & 0 & 3 & 0 & 1 \\
\hline 13 & It is difficult for me to say 'no' to testing when doctors/midwives offer it & 1 & -2 & -3 & -2 & 2 \\
\hline 14 & I would take lots of time to make a decision about testing & -3 & 0 & -3 & 0 & 0 \\
\hline 15 & Having too much information about the tests makes it difficult to make decisions & 0 & 0 & -1 & 1 & -1 \\
\hline 16 & I find it hard to make a decision about testing because there are too many decisions to make in pregnancy & -3 & -1 & 0 & -1 & 1 \\
\hline 17 & I would discuss it with my partner/husband but the decision would be mine. & 2 & 4 & -1 & 0 & 0 \\
\hline 18 & I would not want to go against my partner/husband's wishes, so if we disagree, I would do what he wants & -1 & -1 & 1 & 1 & 0 \\
\hline 19 & Me and my partner/husband should make the decision about testing together & 2 & 3 & 4 & 3 & 4 \\
\hline 20 & I would keep my in-laws out of the process of making the decision about testing & 1 & 1 & -2 & -2 & -1 \\
\hline 21 & I would take advice from my parents or brothers/sisters about having the tests & -1 & -1 & 1 & 1 & 2 \\
\hline 22 & My parents' or brothers'/sisters' views would sway my decision about testing & -2 & -2 & -1 & 1 & 1 \\
\hline 23 & My in-laws' views would influence my decision about testing & -3 & -4 & -2 & 0 & -1 \\
\hline 24 & I think doctor's/midwife's should give information only, not advice about whether to have testing & -2 & 1 & 0 & -1 & -3 \\
\hline 25 & I believe doctors/midwives would not offer the tests if it wasn't important to have them & 3 & 1 & 2 & 2 & 2 \\
\hline 26 & I believe having these tests is just part of being a good mother & 4 & 2 & 3 & 4 & 3 \\
\hline 27 & I would want information provided by doctors/midwives to help me make my decision about testing & 3 & 2 & 4 & 2 & 3 \\
\hline 28 & I would consider myself fortunate to be offered these tests free of charge & 1 & 1 & -1 & 2 & 1 \\
\hline 29 & I would worry about what others might think if I decided to terminate a child & -1 & -2 & -2 & -2 & -3 \\
\hline 30 & I should not be asking the doctor or midwife to make a decision about whether or not I have testing & -1 & 1 & 1 & -3 & 0 \\
\hline 31 & I value the opportunity to think about termination of a child with a condition & 1 & 4 & 2 & 2 & 0 \\
\hline 32 & If I cannot decide whether to have testing then I should not be tested & -1 & 1 & 1 & -2 & -1 \\
\hline 33 & $\begin{array}{l}\text { Decisions about testing should only be made after carefully thinking through all the possible consequences of } \\
\text { testing }\end{array}$ & 0 & 3 & 1 & -1 & 3 \\
\hline 34 & I would worry about people judging me as being irresponsible if I decide not to have testing. & 0 & -1 & 0 & 1 & -1 \\
\hline 35 & I would not have an abortion, so there's no point in having testing & -3 & -3 & -3 & -3 & -2 \\
\hline 36 & I would accept the child that God gives me so there is no reason to have testing & -4 & -4 & 0 & -4 & -4 \\
\hline 37 & I want information about the tests but I do not want to make the decision & -2 & -1 & 0 & -1 & -3 \\
\hline 38 & I do not want information from doctor's/midwife's-I will use my own judgement & -4 & -3 & -2 & -3 & -4 \\
\hline 39 & My partner/husband should make the decision about testing & -2 & 0 & 0 & 2 & -2 \\
\hline 40 & Doctors should tell me what to do, not ask me to make the decision about testing & 2 & -3 & 2 & -1 & -1 \\
\hline 41 & I prefer not to make the decision about testing because I am scared of making the wrong decision & -1 & -2 & 0 & -2 & - \\
\hline
\end{tabular}

similarities within factors and difference between them. Varimax rotation was used to ensure that no Q-sort loaded significantly at the same level on more than one factor [25].
Q-sorts exemplifying each factor (exemplars) were identified. These were Q-sorts with a loading of $+0.4(p<$ 0.01 ) on one factor only [25]. Next, these exemplars were merged to produce a factor array-a single 'ideal' Q-sort 
that best represented each factor (Table 2) [26], by using a weighting formula [27]. These factor arrays represent how an individual with a correlation coefficient of one would have ranked the 41 statements. Therefore, factor arrays represent a particular viewpoint on the research topic and are the key output of the statistical analysis that are then interpreted.

Eight factors were originally extracted, with an eigenvalue of 1.00 or more [27], with a minimum of one exemplar [26]. A five factor solution was reached after inspection of factors 6-8 showed that they did not provide distinct viewpoints that were not captured in the other five factors.

Interpretation of the factors (identifying distinct viewpoints) involved examining and comparing the factor arrays, by focussing on the statements in the 'strongly agree' and 'strongly disagree' columns, and statements identified as statistically distinguishing each factor. Post-sorting interviews were then used to inform, confirm or challenge, and further clarify factor interpretations. SA initially interpreted the factors, discussed them with the co-authors and refined them on a number of occasions until all co-authors agreed the interpretation.

\section{Results}

See Table 3 for demographic information for the exemplars in the five factors (viewpoints). Quotations presented in this section will be followed by participants' codes, which include information about their study location $(\mathrm{SH}=$ Shanghai, $\quad \mathrm{DY}=$ Duyun, $\quad \mathrm{HK}=$ Hong $\quad$ Kong, $\quad \mathrm{PK}=$ Pakistan).

There was one consensus statement across all the factor arrays, where participants tended to strongly agree with doctors giving professional advice about uptake of testing.

\section{Viewpoint 1: choice as a maternal responsibility entrusted to doctors}

Q-sorts of 40 participants exemplified this viewpoint: 9 from Duyun, 10 from Shanghai, 6 from HK and 15 from Pakistan.

In this viewpoint, the emphasis was on being a responsible mother by having all available antenatal tests, and the doctor's role in giving directive advice and guidance was perceived as central. Participants valued antenatal testing and many did not seem to recognise the need to make a decision about testing. Participants tended to agree most strongly with having antenatal tests as part of 'good antenatal care' $(\# 12=+4)$ and 'being a good mother' (\#26 $=+4)$. This was because they believed they had a

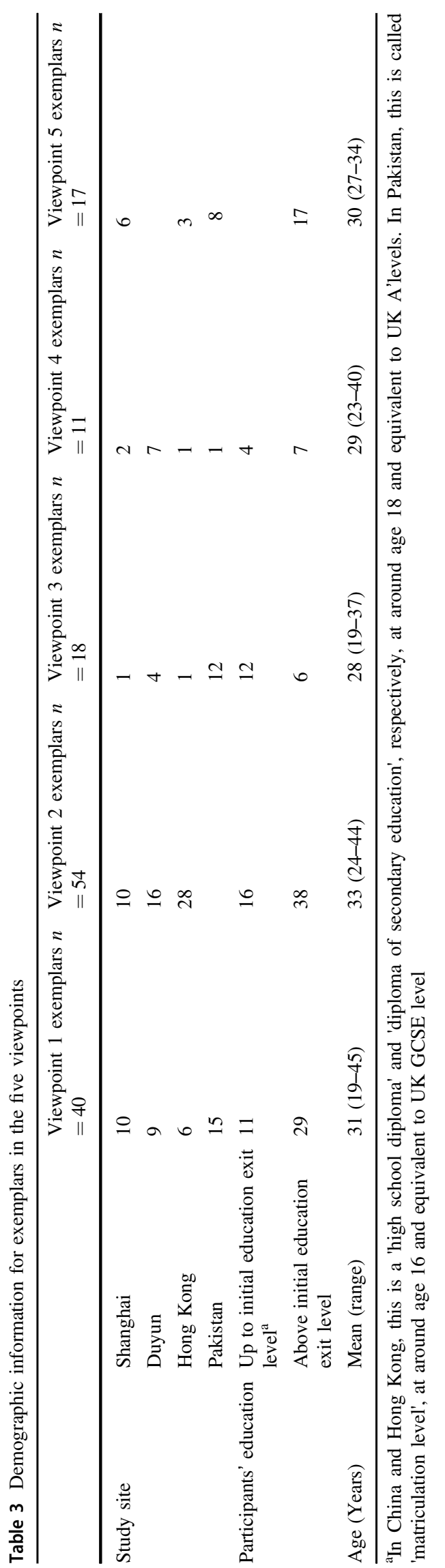


responsibility to themselves, their child, family and society to prevent having a child with a condition:

If I don't have tests, people may think I'm a careless mother. (PK11)

To give birth to this baby is to bring harm to him throughout life. (DY25)

If I can terminate ... it won't become a burden to family and society. (SH08)

Participants' accounts show they highly trusted and valued doctors' professional and directive advice. They strongly disagreed with 'I do not want information from doctors, I will use my own judgement' (\#38=-4), and believed that doctors acted in patients' best interest $(\# 25=+3)$ :

I trust doctors in everything. (HK30); You entrust yourself to doctors. (PK49)

Participants strongly disagreed with accepting "the child that God gives me so there is no reason to have testing" (\#36 = -4), further emphasising the importance antenatal tests. They agreed it was important to think about the challenge of bringing up a child with a condition $(\# 2=+2)$, and to consider options following abnormal test results.

While participant wanted directive advice, they also wanted information $(\# 27=+3)$ and to retain autonomy over the decision about antenatal testing:

The doctor must tell me everything. Whether to accept it or not is up to me. (DY30)

Nevertheless, some participants seemed dependent on doctors to make the decision about antenatal screening due to lack of confidence in their own ability, or were content to play a passive role by leaving the decision to doctors $(\# 9=$ +2 ; $\# 40=+2, \# 30=-1$ ). Accordingly, being tested without permission was not a concern in this viewpoint $(\# 7=0)$ :

I do whatever the doctor requires. (SH94)

Doctors know best... I don't need to think whether it's good for me. (PK04)

Participants in this viewpoint placed little emphasis on religion $(\# 5=0)$.

\section{Viewpoint 2: choice as a shared decision with the partner led by the mother}

Q-sorts of 54 participants exemplified this viewpoint: 16 from Duyun, 10 from Shanghai and 28 from HK.

In contrast to viewpoint 1, participants emphasised the importance of shared decision-making with their partner $(\# 17=+4 ; \# 19=+3)$, explaining that this would enable them to share stress and responsibility of the decision, and prevent isolation:

I can't decide myself. I need to do it with my husband. (GH72)

He can help share the stress... I don't have to be alone. (HK23)

This was also the viewpoint in which participants most strongly emphasised importance of the mother's autonomy over the final decision $(\# 17=+4)$ :

My husband would listen to me. I'd make the final decision. (HK10)

Also in contrast to viewpoint 1 , doctors were viewed as information providers. Participants tended to strongly disagree with leaving the decision to doctors or with doctors telling them what to do $(\# 9=-3 ; \# 40=-3)$, and agree with not asking doctors to make decisions $(\# 30=+1)$ and carefully thinking through the possible consequence of testing $(\# 33=+3)$. Participants' reasons include mistrust of doctors and belief in the rights of the pregnant woman to make autonomous decisions:

I don't trust doctors... I'd never allow them to make a decision for me (DY17)

Doctors ask you to do more examinations for financial gain. (SH03)

Furthermore, participants most strongly agreed with valuing the opportunity to think about termination of a child with a condition ( $\# 31=+4)$, possibly because they would worry about the child being treated badly by society $(\# 4=$ +3 ). Similar to viewpoint 1 , having tests was considered essential $(\# 36=-4 ; \# 35=-3 ; \# 25=+1)$, although the results were believed to enable parents to make reproductive decisions by considering (i) the burden of an affected child and (ii) whether to opt for termination of pregnancy: 
It's unnecessary to keep the baby. It would be a huge burden for him and us. (DY54)

...testing allows parents to decide ways of caring for their affected child. (HK60)

Participants also had strong rejection to involving family members in the decision-making process $(\# 23=-4 ; \# 20=$ +1 ), as the child was considered the parents' responsibility.

\section{Viewpoint 3: choice as a shared decision led by the partner}

Q-sorts of 18 participants exemplified this viewpoint: 12 from Pakistan, 4 from Duyun, 1 from Shanghai and 1 from HK.

Similar to viewpoint 1, participants wanted HCPs to support decision-making about testing $(\# 27=+4)$, although the need for directive advice is less prominent $(\# 7=+2 ; \# 30$ $=+1$ ), and similar to viewpoint 2 , they wanted to make the decisions with their partner $(\# 19=+4)$. However, in contrast to viewpoint 2, participants' would want their partner to take the lead in decision-making. Participants tended to disagree with 'I would discuss it with my partner but the decision would be mine' (\#17 = -1) and agree with 'I would not want to go against my partner's wishes, so if we disagree, I would do what he wants' $(\# 18=+1)$ :

The decision is for us both, although his view takes priority. (PK06)

Another distinct feature of this viewpoint is participants' decisions would be informed by their values of people with disabilities. While participants valued antenatal screening (\#12 $=+3$ ), they were likely to disagree most strongly with worrying 'about the child being treated badly by society' and 'offer of tests suggests that people with these conditions are worth less than others' (\#4=-4; \#3=-4). This value of a child with a condition was unrelated to religious beliefs $(\# 5=-1$; $\# 36$ =0). Instead, participants talked about the importance of accepting and valuing their child irrespective of any condition:

He's your flesh. If you reject him... you don't deserve to be his parents. (DY37)

\section{Viewpoint 4: choice as a responsibility delegated to the partner and doctors}

Q-sorts of 11 participants exemplified this viewpoint: 7 from Duyun, 2 from Shanghai, 1 from HK and 1 from Pakistan.

As in the other viewpoints, participants wanted to make decisions with their partner $(\# 19=+3 ; \# 18=+1)$. In contrast, this was the only account in which some participants agreed that their 'partner should make the decision' $(\# 39=+2)$. Participants would also let doctors make the decision for them $(\# 9=+1 ; \# 30=-3)$, because they valued doctors' professional opinion, although they did not want to be told what to do $(\# 40=-1)$ :

I need to make the decision with my husband, which makes me feel safe. (DY96)

No matter what the doctor decides, I'd listen to him. (DY71)

Participants were most concerned about a child with a condition being treated badly by society $(\# 4=+3)$ and the importance of thinking about the challenges of bring up a child with a condition $(\# 2=+3)$. They were least concerned about 'the offer of the test suggests that people with these conditions are worth less than others' $(\# 3=0)$. Participants' accounts suggest this was because a child with a condition would be considered a burden and, therefore, valued preventive measures. These views also explain participants' value of antenatal testing $(\# 26=+4 ; \# 32=-2)$ :

...baby would be looked down upon, mocked... all parents have the same idea that it's a serious burden for families to raise such children. (DY99)

Another strong theme in this account was that participants were explicitly dismissive of religion, as they most strongly disagreed with looking to religion for guidance about testing $(\# 5=-4)$ and with accepting the child that God gives $(\# 36=-4)$. This may be because most participants in this account did not have religious beliefs and had strong views about the insignificance of religion in decision-making.

\section{Viewpoint 5: choice within a religious discourse}

Q-sorts of 17 participants exemplified this viewpoint: 8 from Pakistan, 6 from Shanghai and 3 from HK.

As in the other viewpoints, participants valued doctors' professional advice $(\# 8=+4 ; \# 24=-3)$, and wanted to make the decision with their partner $(\# 19=+4)$. A distinct feature of this viewpoint is the importance of religion in decision-making, as most participants strongly agreed that they would look for what their religion says about having antenatal tests $(\# 5=+3)$ :

My religious beliefs affect my value of life... it's hard to separate them. (HK07) 
However, as in all the other viewpoints, participants also strongly disagreed with accepting the child that God gives (\#36 = -4). The contradictory placement of these two statements (\#5 and \#36) highlights the importance of religious discourse for these participants, but their religious beliefs would not necessarily deter them from opting for antenatal tests. For example, participants emphasised the importance of considering the pragmatic implication of having a child with a condition and using the knowledge given by God to prevent suffering of both the parents and child:

...it's painful to see the child's suffering... we should think 'what's best for and the child'... and use this knowledge to make life better rather than miserable. (PK51)

Accordingly, participants in this account would most likely opt for termination of pregnancy $(\# 35=-2)$ and not be concerned about what others may think $(\# 29=-3)$ :

If there is any problem, we can take timely intervening measures. (SH51)

Participants in this account also believed that the decision was not theirs alone $(\# 6=-3)$ and that it was important to involve other family members in decision-making (\#21 = +2 ), because of their role in caring for an affected child.

\section{Discussion}

This study elicits five distinct viewpoints about how women from China, $\mathrm{HK}$ and Pakistan perceive informed choice for antenatal screening, showing a range of preferences for decision-making. Women within most of the viewpoints tended to strongly agree with doctors giving professional, even directive, advice about antenatal testing and with making the decision with their partner. Viewpoint 2 aligns closely with a more individualistic viewpoint in a similar UK study [20], where women believed that decisionmaking should be supported but not directed by their partner, although women in the present study placed comparatively little emphasis on individual rights. Overall the findings of the present study suggest the need for adapting relational approaches to autonomy in policy and practice guidelines for antenatal screening.

The viewpoints are not country specific because women from all the study locations were represented in viewpoints 1,3 and 4, and from three locations in viewpoints 2 and 5 . Nevertheless, viewpoints 3 and 5 were more representative of women from Pakistan and viewpoint 2 of women from Chinese populations only, particularly HK. Viewpoint 2 partially resembles an individualistic approach to informed choice, possibly due to influence of Western culture in HK. Furthermore, differences in viewpoints 2 and 5 about the involvement of family members in decision-making suggest cultural differences in family structures, with more interdependence in Pakistani families. Overall, the findings highlight ethical dilemmas for HCPs in facilitating decisionmaking. Policy and practice guidelines globally need to (re) frame decision-making for antenatal screening using relational approaches to autonomy [5, 6].

Similar to previous research [20], women in most viewpoints valued professional advice [28, 29]. Yet research in HK shows HCPs are likely to refrain from involvement in antenatal screening decisions [30]. Support from HCPs can enhance autonomous choices [31], thus, it is essential for them to engage in discussion with women. However, women's need to entrust decisions to doctors (viewpoint 1) raises ethical concerns, particularly in light of doctors' potential conflict of interest between women's clinical needs and income generation through private screening. Our findings, together with technological advances resulting in the constantly increasing number of conditions testable in pregnancy, suggest the need for political, ethical and theoretical debates to clarify the role of HCPs in supporting women's decision-making for antenatal screening to inform the development of culturally appropriate approaches to autonomy. Further research should explore how to adapt relational approaches encapsulating principles of shared decision-making, whilst preventing paternalistic influences on screening decisions.

In contrast to most viewpoints, women in viewpoint 2 mistrusted doctors, believing they were conflicted, recommending tests for financial gain. Given that women in this study, like others [28], often wanted HCPs to facilitate decision-making, further research is needed on how to enhance trust between HCPs and patients in the context of private antenatal screening to ensure adequate support for autonomous decision-making [32].

Similar to others $[18,20]$, our study provides limited support for involving other family members, particularly 'in-laws', in decision-making about antenatal screening. However, the partner's role was generally viewed as central to the decision-making process. The partner's views about termination of pregnancy and fathering a child with a disability are major factors in women's decision-making [33]. Women may also want to share responsibility for any adverse outcomes; want emotional and financial support; and support to seek/synthesise antenatal screening information [18, 20, 33]. There is little reference to partners in screening guidelines in any country [33]. Our findings highlight the need for clarification of the role of the partner 
from policy and clinical practice perspectives, particularly how HCPs should involve partners in decision-making. Furthermore, research is needed on how such shared decision-making could be facilitated using relational approaches, while ensuring the absence of undue influences from HCPs and others on the woman's decision.

Our findings also suggest that decisions about antenatal screening may take place within a religious discourse, but this should not be interpreted as women would reject antenatal screening. Instead, women are likely to focus on the implications of the condition for the child and issues related with raising the child [34]. HCPs should focus on information provision to support women's decision-making. The availability of such information is limited in Asian countries and needs further research and development.

Throughout the study, women referred to the social norm of preventing a child becoming a burden on society, and being a responsible mother by opting for all antenatal screening tests. In China and HK, these findings may be due to the use of the concept 'Yousheng' meaning 'healthy birth', which is related to the social goal of reducing the incidence of birth defects to improve population quality. In China, during public education classes for pregnant couples, birth defects are associated with poor quality of life and poor quality of population, and economic consequences of having an affected child are highlighted, hence stigmatising children with genetic conditions, promoting the use of antenatal screening [35]. Also, Medical, educational and social welfare facilities for people with disabilities are lacking, so the responsibility for educating, supporting and caring for a child with a disability resides entirely with the family [36]. Our findings show that parents may value their child irrespective of any conditions or concerns about social stigma, so may not necessarily want to opt for antenatal screening to ensure a 'healthy birth' but, similar to other populations, may feel pressure to do so [37]. In Chinese Maternal and Infant Law, the rights of parents to decide to have a child with a genetic condition are acknowledged. However, changes are needed in practice to ensure these pressures do not have undue influence on parents' screening decisions, instead ensuring prospective parents are supported to make decisions about antenatal screening in accordance with their own values and beliefs [4].

\section{Study limitations}

Some viewpoints were held by comparatively smaller numbers of participants. As a qualitative approach, Qmethodology enables claims about the diversity of viewpoints in a sample, not the percentage of the sample representing these viewpoints or their prevalence in the general population. Therefore, claims about differences between the study locations in perceptions of informed choice are tentative. The prevalence of these viewpoints within the study locations needs further research. Also, as our first Q-methodology study in Asia, the focus was on women's views. Plus, we encountered difficulties in recruiting participants with a lower educational exit level, and acknowledge that a more diverse sample by educational attainment may have resulted in more diverse viewpoints. Further research should include exploration of men's and HCPs' views, and ensure diversity by educational attainment.

\section{Conclusion}

This study identified five distinct viewpoints about 'informed choice' across China, HK and Pakistan, highlighting ethical dilemmas for HCPs in implementing antenatal screening. Women's preferences for decisionmaking with HCPs and/or their partner, with minimal emphasise on individual rights, suggest the need for clarification of the role of HCPs in supporting and facilitating decision-making to enhance women's autonomy. Policy and practice guidelines need to be (re)framed to facilitate decision-making processes for antenatal screening using relational approaches to autonomy.

Acknowledgements This research was funded by: the University of Leeds; The Chinese University of Hong Kong; Hong Kong Baptist University; and Central Park Medical College, Lahore.

\section{Compliance with ethical standards}

Conflict of interest The authors declare that they have no conflict of interest.

\section{References}

1. Van den Veyver IB. Recent advances in prenatal genetic screening and testing. F1000Res. 2016;5:2591.

2. World Health Organization. Medical genetic services in developing countries: the ethical, legal and social implications of genetic testing and screening. 2006, http://www.who.int/ genomics/publications/GTS-MedicalGeneticServices-oct06.pdf. Accessed 8 Aug 2017.

3. Beauchamp TL, Childress JF. Principles of biomedical ethics, 5th ed. Oxford: Oxford University Press; 2001.

4. Bekker H, Thornton JG, Airey CM, et al. Informed decision making: an annotated bibliography and systematic review. Health Technol Assess. 1999;3:1-156.

5. Kim SYH. Autonomy and the relational self. Philos Psychiatr Psychol. 2013;20:183-5.

6. Turoldo F. Relational autonomy and multiculturalism. Camb Q Healthc Ethics. 2010;19:542-9.

7. Mackenzie C, Stolijar N. Feminist perspectives on automony, agency, and the social self. Oxford: Oxford University Press; 2000.

8. Evans M, Bergum V, Bamforth S, MacPhail S. Relational ethics and genetic counseling. Nurs Ethics. 2004;11:459-71. 
9. Entwistle VA, Carter SM, Cribb A, McCaffery K. Supporting patient autonomy: the importance of clinician-patient relationships. J Gen Intern Med. 2010;25:741-5.

10. Samuel GN, Dheensa S, Farsides B, Fenwick A, Lucassen A. Healthcare professionals' and patients' perspectives on consent to clinical genetic testing: moving towards a more relational approach. BMC Med Ethics. 2017;18:47.

11. van den Heuvel A, Chitty L, Dormandy E, et al. Is informed choice in prenatal testing universally valued? A population-based survey in Europe and Asia. BJOG. 2009;116:880-5.

12. Chandrasekharan S, Minear MA, Hung A, Allyse $M$. Noninvasive prenatal testing goes global. Sci Transl Med. 2014;6:231fs 215 .

13. Ho A. Relational autonomy or undue pressure? Family's role in medical decision-making. Scand J Caring Sci. 2008;22:128-35.

14. Moazam F. Families, patients, and physicians in medical decision making: a Pakistani perspective. Hastings Cent Rep. 2000;30:28-37.

15. Bowman KW, Hui EC. Bioethics for clinicians: 20. Chinese bioethics. CMAJ . 2000;163:1481-5.

16. Tamura $\mathrm{C}$. The family-facilitated approach could be dangerous if there is pressure by family dynamics. Am J Bioeth. 2006;6:16-18.

17. Mumtaz Z, Salway S. Understanding gendered influences on women's reproductive health in Pakistan: moving beyond the autonomy paradigm. Soc Sci Med. 2009;68:1349-56.

18. Lau JY, Yi H, Ahmed S. Decision-making for non-invasive prenatal testing for Down syndrome: Hong Kong Chinese women's preferences for individual vs relational autonomy. Clin Genet. 2016;89:550-6.

19. Mozersky J, Ravitsky V, Rapp R, Michie M, Chandrasekharan S, Allyse M. Toward an ethically sensitive implementation of noninvasive prenatal screening in the global context. Hastings Cent Rep. 2017;47:41-49.

20. Ahmed S, Bryant LD, Tizro Z, Shickle D. Interpretations of informed choice in antenatal screening: a cross-cultural, Qmethodology study. Soc Sci Med. 2012;74:997-1004.

21. van Exel J, Baker R, Mason H, Donaldson C, Brouwer W. Public views on principles for health care priority setting: findings of a European cross-country study using Q methodology. Soc Sci Med. 2015;126:128-37.

22. Brown SQ. Methodology and qualitative research. Qual Health Res. 1996;4:561-7.
23. Kitzinger C. The Social Construct of Lesbianism. Bristol: Sage; 1987.

24. Schmolck P PQ Method Download Page for Windows Users (version 2.35). 2014, http://schmolck.userweb.mwn.de/qmethod/ downpqwin.htm. Accessed 8 Aug 2017.

25. Watts S, Stenner P. Doing Q methodology: theory, method, and interpretation. Qual Res Psychol. 2005;2:67-91.

26. Stenner PH, Cooper D, Skevington SM. Putting the Q into quality of life; the identification of subjective constructions of healthrelated quality of life using Q methodology. Soc Sci Med. 2003;57:2161-72.

27. Brown SR. Political Subjectivity: applications of Q methodology in political science. New Haven: Yale University Press; 1980.

28. Ahmed S, Bryant LD, Tizro Z, Shickle D. Is advice incompatible with autonomous informed choice? Women's perceptions of advice in the context of antenatal screening: a qualitative study. Health Expect. 2012;10:7625.

29. Deber RB, Kraetschmer N, Irvine J. What role do patients wish to play in treatment decision making? Arch Intern Med. 1996;156:1414-20.

30. Pilnick A, Zayts O. Advice, authority and autonomy in shared decision-making in antenatal screening: the importance of context. Sociol Health Illn. 2016;38:343-59.

31. Quill TE, Brody H. Physician recommendations and patient autonomy: finding a balance between physician power and patient choice. Ann Intern Med. 1996;125:763-9.

32. Grady C. Enduring and emerging challenges of informed consent. N Engl J Med. 2015;372:855-62.

33. Dheensa S, Metcalfe A, Williams RA. Men's experiences of antenatal screening: a metasynthesis of the qualitative research. Int J Nurs Stud. 2013;50:121-33.

34. Bryant LD, Ahmed S, Ahmed M, Jafri H, Raashid Y. 'All is done by Allah'. Understandings of Down syndrome and prenatal testing in Pakistan. Soc Sci Med. 2011;72:1393-9.

35. Zhu J. Projecting potentiality: understanding maternal serum screening in contemporary China. Curr Anthropol. 2013;54: S36-44.

36. Shen J. Precision assessment of public attitudes toward genetic testing. Am J Med Genet A. 2016;170:3185-8.

37. Deans Z, Hill M, Chitty LS, Lewis C. Non-invasive prenatal testing for single gene disorders: exploring the ethics. Eur J Hum Genet. 2013;21:713-8. 